\title{
RESEARCH HIGHLIGHTS
}

NEURODEGENERATION

\section{Parkinson's propagator}

The brains of patients with Parkinson's disease are riddled with clumps of misfolded a-synuclein protein, which is normally soluble in brain cells. Virginia Lee and her colleagues at the University of Pennsylvania in Philadelphia show that synthetic fibrils of this protein can enter mouse neurons unassisted and convert normal $\alpha$-synuclein into the insoluble aggregates seen in human brains.

Two weeks after mixing the fibrils with the neurons, the authors observed an increase in insoluble clumps and a decrease in normal $\alpha$-synuclein in the cells. The clumps spread throughout the neurons, impairing their ability to form connections. Cell death was $68 \%$ higher in treated cells than in control neurons.

The findings suggest a mechanism for how neurodegeneration can 'spread' throughout the brain. Neuron 72, 57-71 (2011)

\section{NANOTECHNOLOGY}

\section{Sugar and shake sensor power}

Tiny sensors implanted into the body for medical monitoring can be powered in one of two ways: by metabolizing surrounding sugar or by harvesting the mechanical energy generated by small vibrations. A group

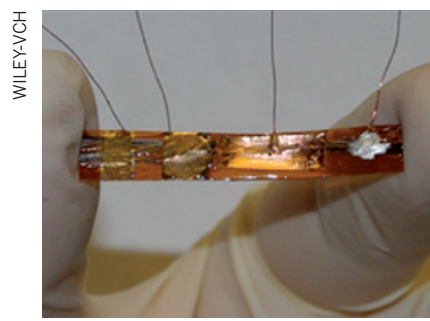

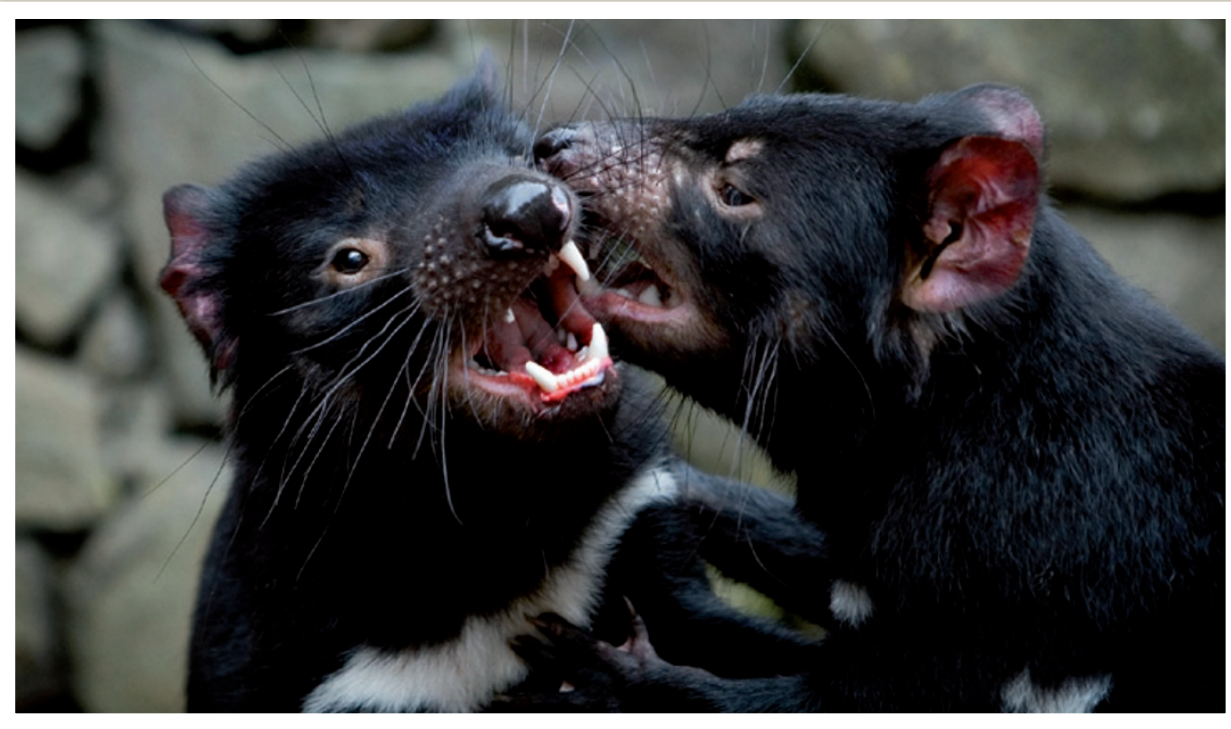

CONSERVATION BIOLOGY

\section{Culls won't save Tasmanian devils}

Killing off Tasmanian devils that are infected with a contagious and deadly facial cancer is unlikely to control the disease.

The cancer, which is thought to be spread through biting, is threatening the Tasmanian devil (Sarcophilus harrisii; pictured) with extinction. Wildlife managers have tried culling infected animals in an isolated population, but to no avail. Nick Beeton at the University of Tasmania in Hobart and Hamish McCallum of Griffith University in Nathan, Australia, have modelled the disease's transmission and various culling strategies. They report that, in a typical population, as many as $96 \%$ would have to be removed on a continuous basis to save the population and wipe out the disease. Such a high removal rate isn't achievable because some animals - more than $20 \%$ at one trial site avoid traps.

Other strategies include keeping a population of uninfected animals in zoos and reserves, and breeding naturally resistant animals.

J. Appl. Ecol. http://dx.doi.org/10.1111/j.13652664.2011.02060.x (2011) led by Zhong Lin Wang of the Georgia Institute of Technology in Atlanta now describes a device (pictured) that can harvest energy from either source, or both at once.

The carbon fibre carries a biofuel cell on one end that uses immobilized enzymes to break down glucose and generate current. The other end is coated with a zinc oxide film, which generates current when force is applied. The researchers made measurements on a bundle of about 1,000 such fibres.

The hybrid generator can also be used to detect pressure variation in biological liquids such as blood, the researchers suggest.

Angew. Chem. http://dx.doi. org/10.1002/anie.201104197 (2011)

\section{CLIMATE-CHANGE ECOLOGY}

\section{Foreshadows of climates past}

Animals with small ranges and those living in regions that have not experienced major climate change in the recent geological past face an increased risk of extinction as the planet warms.

Brody Sandel at Aarhus University in Denmark and his colleagues compared the speed of climate change in various regions during the past 21,000 years with patterns in the numbers and range sizes of mammals, birds and amphibians endemic in those regions. They found associations between rapid historical climate fluctuations and a low number of endemic species in Europe and northeastern North America. By contrast, the highest 